\title{
Psychometric Properties of the Traditional Chinese Version of the Child and Adolescent Needs and Strengths-Trauma Comprehensive
}

\author{
KC Yau, SM Chan
}

\begin{abstract}
Objective: To determine the internal consistency, construct validity, and scaling properties of the traditional Chinese version of the Child and Adolescent Needs and Strengths-Trauma Comprehensive (TC-CANS-Trauma).

Methods: 66 male and 62 female children, adolescents, and young adults aged 3 to 22 years who were referred to trauma treatment service were selected by convenience sampling. The original English version of the CANS-Trauma was translated to traditional Chinese by a medical professional, back-translated to English by a clinical psychologist, and then cross-checked by another psychologist to ensure consistency. Chinese wordings were adjusted to maintain the conceptual rather than literal meaning. Participants were assessed using the TC-CANS-Trauma as well as the traditional Chinese version of the Life Events Checklist (LEC), the Children's Impact of Event Scale-Revised (CHIES-R), the Strengths and Difficulties Questionnaire-Impact Component (SDQ-Impact), and the Parenting Sense of Competence (PSOC). Internal consistency of eight primary domains of the TC-CANS-Trauma was evaluated by Cronbach's alpha. Construct (convergent and divergent) validity of five of these domains with the LEC, the CHIES$\mathrm{R}$, the SDQ-Impact, and the PSOC was assessed. Rasch modelling was used to evaluate the scaling properties of the eight primary domains of the TC-CANS-Trauma.

Results: Internal consistency of the eight primary domains of the TC-CANS-Trauma was satisfactory, with Cronbach's alpha ranging from 0.63 to 0.90 . Construct (convergent and divergent) validity of five of these domains with the LEC, the CHIES-R, the SDQ-Impact, and the PSOC was good. In Rasch modelling, most TC-CANS-Trauma domains showed good item separation values. Infit and outfit statistics of most domain items were $<2$ indicating good item fitness in their respective domains. For person separation, all domains of the TC-CANS-Trauma did not have a sufficient discriminability to identify high and low performers.

Conclusions: The TC-CANS-Trauma is valid for comprehensive assessment of trauma-related domains among Hong Kong children and adolescents. Its ratings can be used to guide the levels of clinical intervention required. Clinicians are recommended to implement the TC-CANS-Trauma to facilitate trauma-informed practice in Hong Kong.
\end{abstract}

Key words: Adolescent; Child; Child, preschool; Psychological trauma; Psychometrics; Young adult

King-Chi Yau, Jockey Club Trauma Treatment Service for Children, The Boys' \& Girls' Clubs Association of Hong Kong

Siu-Mei Chan, Jockey Club Trauma Treatment Service for Children, The Boys' \& Girls' Clubs Association of Hong Kong

Address for correspondence: King-Chi Yau, Jockey Club Trauma Treatment Service for Children, The Boys' \& Girls' Clubs Association of Hong Kong, 6/F, 3 Lockhart Road, Wanchai, Hong Kong.

Email:sm.chan@bgca.org.hk

Submitted: 22 October 2019; Accepted: 18 March 2021

\section{Introduction}

Trauma-informed practice involves (1) practitioners' sensitivity towards the effects of trauma, (2) clients' physiological and psychological safety, and (3) service users' strengths and resources. ${ }^{1}$ Service providers should ensure that professional and administrative staff have a comprehensive understanding of the impacts of trauma and the ability to carry out trauma-informed assessment and formulate responsive interventions.,3 Traumatised children tend to have more self-regulation problems, poorer responses to consequences of aggressive behaviours, and more deficient of optimism. ${ }^{4}$ Complex trauma (ie, prolonged exposure to adverse interpersonal events such as abuse) can be detrimental to children's development ${ }^{5}$ and may result in developmental difficulties such as insecure attachment, affect dysregulation, and cognitive dysfunction. ${ }^{6}$ The array of needs of traumatised children necessitates the traumainformed practice among frontline healthcare service providers.

From 2001 to 2010, the incidence of child abuse in Hong Kong had increased by $87 \%$. The rate of admission to hospital secondary to child maltreatment has increased since 2001. ${ }^{7}$ Hong Kong children with experiences of complex trauma are associated with a higher degree of 
attachment insecurity and posttraumatic stress disorder reactions. ${ }^{8}$ A Hong Kong commissioned report concluded an association between child abuse and mental illness among victims. ${ }^{7}$ Indeed, exposure to abuse affects both victims and perpetrators. $66 \%$ of perpetrators of child abuse have a history of child maltreatment themselves; this suggests an intergenerational transmission of trauma. ${ }^{7}$ The prevalence of depressive and anxiety disorders among Hong Kong adults is $13.3 \%$, which is comparable to that in other developed regions, ${ }^{9}$ whereas the prevalence of common mental disorders among Hong Kong adolescents is $16.4 \% .{ }^{10}$ In Hong Kong, the number of patients seeking public hospitals' psychiatric services increased from 187000 in 2011-12 to $>220000$ in 2015-16. ${ }^{11}$ Thus, service providers need to equip their frontline staff with higher sensitivity to trauma among children and adolescents, as well as caregivers. However, trauma-informed practice is not yet a mainstream practice in Hong Kong. ${ }^{12}$ Validation studies in Hong Kong mainly focus on tools for screening and identifying trauma events or symptoms, ${ }^{13-15}$ rather than comprehensive trauma-informed assessment. Therefore, the aim of the present study was to determine the internal consistency, construct validity, and scaling properties of the traditional Chinese version of the Child and Adolescent Needs and Strengths-Trauma Comprehensive (TC-CANSTrauma).

\section{Methods}

66 male and 62 female children, adolescents, and young adults aged 3 to 22 (mean, 10.75 \pm 4.89 ) years who were referred to trauma treatment service by a non-governmental organisation were selected by convenience sampling. Informed consent was obtained from each participant; participants were free to withdraw from the study at any time. Caregivers of the participants were most commonly mothers $(80.5 \%)$, followed by fathers $(12.5 \%)$ and others (7\%) such as grandparents, aunts, and foster care parents.

The original English version of CANS-Trauma was developed by Northwestern University and the National Child Traumatic Stress Network, with its copyrights held by the Praed Foundation for free usage. ${ }^{16}$ It is used to assist in planning and evaluating trauma cases. ${ }^{16}$ It also guides the degree of corresponding interventions required in trauma-informed practice..$^{16}$ It is a 110 -item, clinician-rated tool that covers eight primary domains: trauma exposure, traumatic stress symptoms, child strengths, life functioning, acculturation, child emotional/behavioural needs (ie, child mental health), child risk behaviours, and caregiver needs and strengths. In addition, there are two optional domains. Each item is rated on a 4-point scale from 0 (no evidence of trauma, no evidence of need, or centrepiece strength), 1 (single incident, mild need, or useful strength), 2 (multiple incidents, moderate need, or identified strength but must be built) to 3 (repeated incidents, severe need, or no strength). Higher scores indicate greater needs and less strength. The ratings for the scale guide the levels of action: 0 (no need for action), 1 (prevention), 2 (action needed), and 3 (immediate action); ratings of 2 and 3 require clinical interventions. ${ }^{16}$ The original CANS-Trauma has acceptable internal consistency and good inter-rater reliability, convergent validity, and scaling properties. ${ }^{17}$ With reference to previous studies, ${ }^{18-20}$ the TC-CANS-Trauma is expected to demonstrate at least acceptable internal consistency and convergent validity.

The original English version was translated to traditional Chinese by a medical professional, backtranslated to English by a clinical psychologist, and then cross-checked by another psychologist to ensure consistency. Chinese wordings were adjusted to maintain the conceptual rather than literal meaning. The TC-CANSTrauma was then used by trained clinicians for clients and caregivers. The TC-CANS-Trauma was completed by clinicians who had been trained through the tool's training website. Trainees needed to rate a clinical vignette to pass a test with a reliability score of $\geq 0.70$.

Other tools used included the traditional Chinese version of the Life Events Checklist (LEC), Children's Impact of Event Scale-Revised (CHIES-R), Strengths and Difficulties Questionnaire-Impact Component (SDQImpact), and Parenting Sense of Competence (PSOC).

The LEC is a 17-item clinician-administered structured interview to assess respondent's exposure to traumatic events, with ratings from 0 (does not apply), 1 (not sure), 2 (learned about it), 3 (witnessed it) to 4 (happened to me)..$^{21,22}$ The original LEC has optimal convergent validity. ${ }^{23}$ The Traditional Chinese version of the clinician-administered posttraumatic stress disorder scale for DSM-IV has been validated against the Impact of Event Scale-Revised and the Posttraumatic Stress Disorder Checklist. ${ }^{24}$

The CHIES-R is a 15 -item, self-report scale that measures three areas of psychological impacts of trauma (hyperarousal, avoidance, and intrusion) in children. The level of distress for each item is rated on a 5-point scale from 0 (no influence) to 3 (moderate influence) to 5 (greatest influence).$^{25,26}$ The Chinese versions of the CHIES-R have good reliability and construct validity and have a stable three-factor structure. ${ }^{26,27}$

The SDQ comprises 25 behavioural questions rated by a parent or teacher to assess the child's total difficulties (ie, SDQ-Difficulties), SDQ-Impact (social impact in areas of family life, leisure activities, academic learning, and social relationships), and pro-social behaviours. ${ }^{28}$ Ratings range from 0 (none or minimal), 1 (somewhat) to 2 (definite). The Chinese version of SDQ-Difficulties and SDQ-Impact have optimal internal consistency, test-retest reliability, and validity. ${ }^{29,30}$ Compared with the SDQ-Difficulties, the SDQImpact is more sensitive in predicting clinical caseness in the local context. ${ }^{30}$ Thus, the SDQ-Impact was used.

The PSOC is a 17-item, self-rated measure to assess the parents' perception of their abilities to handle parenting in terms of satisfaction and efficacy on a 7-point scale from 1 (strongly disagree) to 7 (strongly agree). ${ }^{31-33}$ The subscale of parenting efficacy was used owing to its higher degree of relevance to the caregiver needs and to the strengths 
domain of the CANS-Trauma. The traditional Chinese version of PSOC has good internal consistency, test-retest reliability, and construct validity, as well as a stable twofactor structure. ${ }^{34}$

Internal consistency of the eight primary domains of the TC-CANS-Trauma was evaluated using Cronbach's alpha. A reliability score of $>0.7$ indicates good internal consistency. A previous psychometric study of the CANSTrauma assessed the convergent and divergent validity of only four domains (trauma exposure, traumatic stress symptoms, child emotional/behavioural needs, and child risk behaviours) based on their expected correlations with other validated tools. ${ }^{17}$ The present study thus assessed convergent and divergent validity of these domains plus the caregiver needs and strengths domain with the LEC, the CHIES-R, the SDQ-Impact, and the PSOC. A Pearson correlation coefficient of $\geq 0.3$ with $p<0.05$ was considered moderate to strong validity. Rasch modelling was used to evaluate the scaling properties of the eight primary domains of the TC-CANS-Trauma. ${ }^{35}$ An item separation value of $\geq 2$, corresponding to an item reliability of $\geq 0.8$, indicates a large enough sample size and wide enough difficulty coverage of the items within the domain. Infit and outfit statistics of $<2$ indicates a good fitness of the items within a domain. A person separation value of $\geq 2$, corresponding to a person reliability of $\geq 0.8$, indicates a good-enough discriminability of the domains to identify high and low scorers..$^{35}$

\section{Results}

Internal consistency of the eight primary domains of the TC-CANS-Trauma was satisfactory, with Cronbach's alpha of 0.90 for child strengths ( 11 items), 0.77 for acculturation (4 items), 0.77 for caregiver needs and strengths (13 items), 0.72 for traumatic stress symptoms ( 8 items), 0.71 for life functioning (13 items), 0.65 for child emotional/behavioural needs (13 items), 0.64 for child risk behaviours (11 items), and 0.63 for trauma exposure (14 items).

The trauma exposure domain was strongly correlated with the LEC $(r=0.66, \mathrm{p}<0.001)$ and weakly correlated with the SDQ-Impact $(r=0.24, \mathrm{p}=0.024)$ but was not correlated with the CHIES-R or the PSOC (Table 1). The traumatic stress symptoms domain was moderately correlated with the LEC $(r=0.35, \mathrm{p}=0.023)$ and the CHIES-R $(r=0.30$, $\mathrm{p}=0.037)$ and weakly correlated with the SDQ-Impact ( $r=0.23, \mathrm{p}=0.035)$ but not correlated with the PSOC. The association between trauma exposure and traumatic stress symptoms leads to the adoption of the posttraumatic stress disorder diagnosis, in which theoretical formulation can be traced back to the stress response syndromes. ${ }^{36}$ The child emotional/behavioural needs domain was moderately associated with the SDQ-Impact $(r=0.39, \mathrm{p}<0.001)$ but was not associated with the LEC, the CHIES-R, or the PSOC. The child risk behaviours domain was moderately associated with the LEC $(r=0.34, \mathrm{p}=0.030)$ and the SDQImpact $(r=0.30, \mathrm{p}=0.007)$ but was not correlated with the CHIES-R or the PSOC. The association between trauma exposure and child risk behaviours has been reported. ${ }^{37,38}$ The caregiver needs and strengths domain was moderately and negatively correlated with the PSOC $(r=-0.31, \mathrm{p}=$ 0.003 ) but was not correlated with the LEC, the CHIES-R, or the SDQ-Impact.

In Rasch modelling, most TC-CANS-Trauma domains showed good item separation values (Table 2), which indicated a sufficient sample size and item difficulty coverage. However, the acculturation domain and the child risk behaviours domain did not have satisfactory item distributions, owing to a narrow item difficulty range in these two domains. With reference to a similar validation study, ${ }^{17}$ the category probability curve of the acculturation domain revealed a lack of endorsement of 3 (ie, severe need) by raters, whereas scores of 0,1 , and 2 were clearly

Table 1. Construct validity between five domains of the traditional Chinese version of the Child and Adolescent Needs and Strengths-Trauma Comprehensive (TC-CANS-Trauma) and other tools

\begin{tabular}{|lccccc|}
\hline Tool & \multicolumn{3}{c|}{ TC-CANS-Trauma domain } \\
\cline { 2 - 6 } & $\begin{array}{c}\text { Trauma } \\
\text { exposure }\end{array}$ & $\begin{array}{c}\text { Traumatic } \\
\text { stress } \\
\text { symptoms }\end{array}$ & $\begin{array}{c}\text { Child } \\
\text { emotional/ } \\
\text { behavioural } \\
\text { needs }\end{array}$ & $\begin{array}{c}\text { Child risk } \\
\text { behaviours }\end{array}$ & $\begin{array}{c}\text { Caregiver } \\
\text { needs and } \\
\text { strengths }\end{array}$ \\
Life Events Checklist & $r=0.66$, & $r=0.35$, & $r=0.28$, & $r=0.34$, & $r=0.20$, \\
& $\mathrm{p}<0.001$ & $\mathrm{p}=0.023$ & $\mathrm{p}=0.073$ & $\mathrm{p}=0.030 \quad \mathrm{p}=0.234$ \\
\hline Children's Impact of Event Scale- & $r=0.05$, & $r=0.30$, & $r=0.11$, & $r=0.18$, & $r=0.07$, \\
Revised & $\mathrm{p}=0.717$ & $\mathrm{p}=0.037$ & $\mathrm{p}=0.442$ & $\mathrm{p}=0.201$ & $\mathrm{p}=0.610$ \\
\hline Strengths and Difficulties & $r=0.24$, & $r=0.23$, & $r=0.39$, & $r=0.30$, & $r=0.01$, \\
Questionnaire-Impact Component & $\mathrm{p}=0.024$ & $\mathrm{p}=0.035$ & $\mathrm{p}<0.001$ & $\mathrm{p}=0.007$ & $\mathrm{p}=0.906$ \\
\hline Parenting Sense of Competence & $r=-0.14$, & $r=-0.13$, & $r=-0.08$, & $r=-0.11$, & $r=-0.31$, \\
& $\mathrm{p}=0.186$ & $\mathrm{p}=0.234$ & $\mathrm{p}=0.461$ & $\mathrm{p}=0.312$ & $\mathrm{p}=0.003$ \\
\hline
\end{tabular}


Table 2.Person and item distribution scores for the eight primary domains of the traditional Chinese version of the Child and Adolescent Needs and Strengths-Trauma Comprehensive (TC-CANS-Trauma)

\begin{tabular}{|lccccc|}
\hline \multirow{2}{*}{ TC-CANS-Trauma domain } & \multicolumn{2}{c}{ Person distribution } & & \multicolumn{2}{c|}{ Item distribution } \\
\cline { 2 - 3 } \cline { 5 - 6 } Trauma exposure & Separation & Reliability & & Separation & Reliability \\
\hline Traumatic stress symptoms & 0.42 & 0.15 & & 2.20 & 0.83 \\
\hline Child strengths & 1.23 & 0.60 & 5.22 & 0.96 \\
\hline Life functioning & 1.59 & 0.72 & 2.90 & 0.89 \\
\hline Acculturation & 1.17 & 0.58 & 2.82 & 0.89 \\
\hline Child emotional/behavioural needs & 0.00 & 0.00 & 0.73 & 0.35 \\
\hline Child risk behaviours & 1.01 & 0.50 & 2.76 & 0.88 \\
\hline Caregiver needs and strengths & 0.00 & 0.00 & 0.32 & 0.09 \\
\hline
\end{tabular}
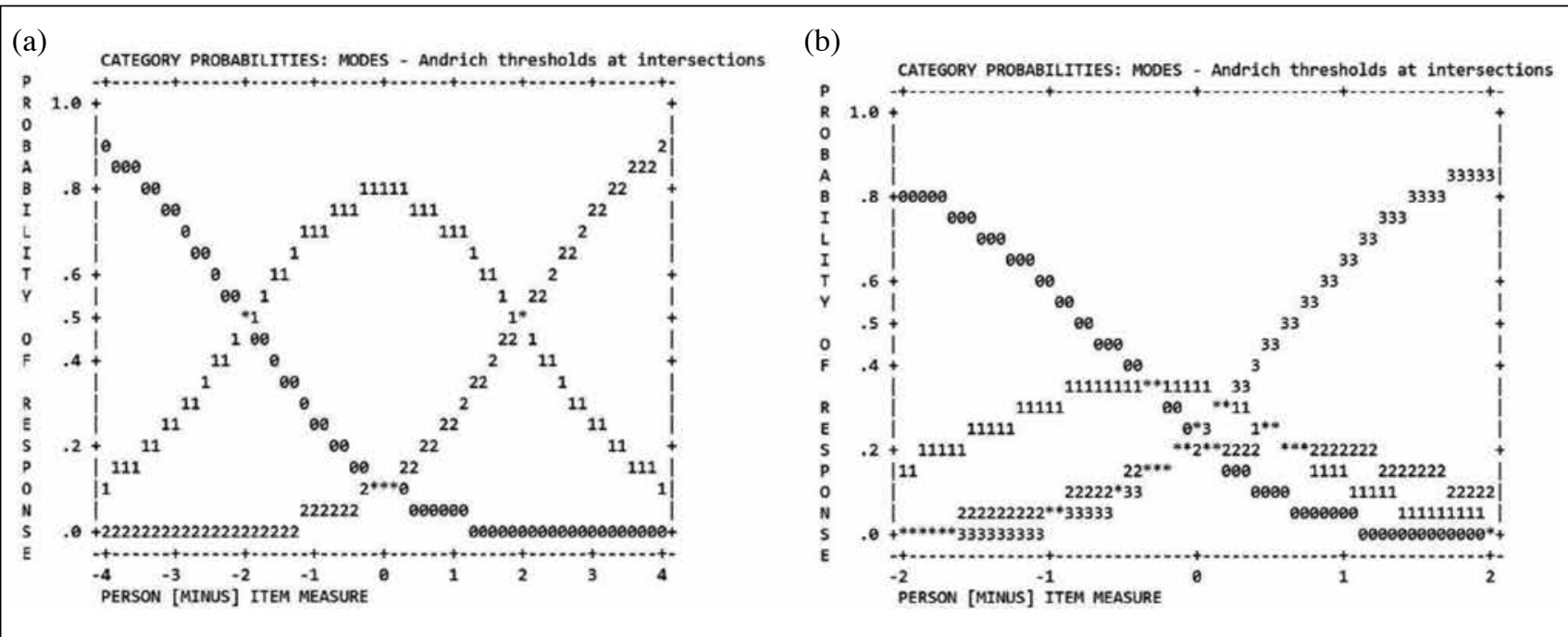

Figure 1. Category probability curves of (a) the acculturation domain and (b) the child risk behaviours domain.

separated (Figure 1a). The category probability curve of the child risk behaviours domain revealed a score of 2 (ie, moderate need), which was not very distinct from other ratings (Figure 1b).

Infit and outfit statistics of most domain items (except for the spiritual/religious item under the child strengths domain, the sexual development item under the life functioning domain, and the marital/partner violence item under the caregiver needs and strengths domain) were $<2$ indicating good item fitness in their respective domains. Thus, each domain measured a construct with a single underlying dimension.

For person separation, all domains of the TC-CANSTrauma did not have sufficient discriminability to identify high and low performers. Thus, the TC-CANS-Trauma was not sensitive enough to differentiate children with high prevalence of symptoms from those with low prevalence of symptoms. The variable maps of all domains consistently showed a low tendency for clinicians to rate a high score (Figure 2). ${ }^{17}$

\section{Discussion}

TC-CANS-Trauma had satisfactory internal consistency across five primary domains, good construct validity, and acceptable scaling properties. The domains of trauma exposure, child emotional/behavioural needs, and child risk behaviours showed marginally acceptable internal consistency. Although removal of some items may slightly increase the reliability, this may compromise the communication value of the assessment tool, ${ }^{19,20}$ because various items in each domain are designed to tap into a 


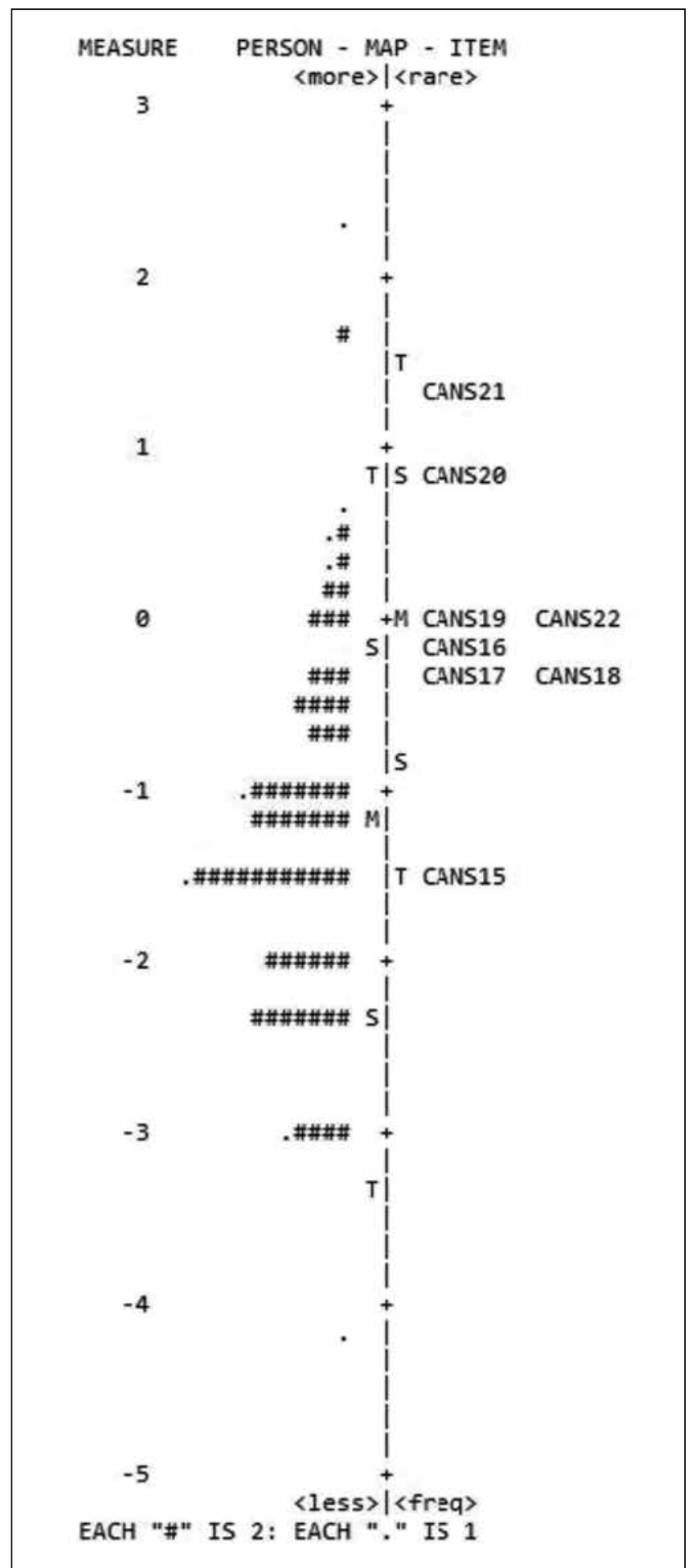

Figure 2. Variable map of the traumatic stress symptoms domain as an example to show a low tendency for clinicians to rate a high score.

wide range of potential needs (eg, suicide risk, sexual aggression, and fire-setting in the child risk behaviours domain). Although a traumatised child or youth is not likely to have elevated scores in all these items, keeping these items in their respective domains is necessary for a comprehensive trauma-informed assessment. Therefore, this study recommends that the TC-CANS-Trauma retain all domain items, owing to the satisfactory internal reliability in general.

Convergent validity of the TC-CANS-Trauma was good. The domains of trauma exposure, traumatic stress symptoms, and caregiver needs and strength were moderately to strongly correlated with the LEC, the CHIES$\mathrm{R}$, and the PSOC, respectively, whereas the domains of child emotional/behavioural needs and child risk were moderately correlated with the SDQ-Impact. Divergent validity of the TC-CANS-Trauma was good. The domains of trauma exposure, child emotional/behavioural needs, and caregiver needs and strengths were weakly or not correlated with constructs of other tools that did not correspond to these three domains. The domains of traumatic stress symptoms and child risk behaviours were moderately associated with the LEC (which measures trauma exposure). Trauma experience increases the risk of having traumatic stress symptoms such as avoidance and risk behaviours such as risky sex. ${ }^{36-38}$

The overall scaling properties of the TC-CANSTrauma were acceptable. The eight primary domains showed sufficient item fitness, and hence they measured constructs with a single underlying dimension. Six of the eight domains demonstrated sufficient difficulty coverage of items. Although the item separation value of the acculturation domain was not satisfactory, this may be due to an absence of such needs in the sample. ${ }^{17,20}$ For the inadequate item separation in the child risk behaviour domain, the less distinctiveness of the score of 2 (ie, moderate need) might indicate that a 3-point scale rather than the current 4-point scale is more adequate for this domain. However, to maintain a consistent scoring system for the TC-CANS-Trauma, the 4-point scale is still recommended for the child risk behaviours domain. A larger sample is needed to generate a more satisfactory item reliability result, particularly for the child risk behaviour domain. ${ }^{35}$

All domains of the TC-CANS-Trauma were less able to discriminate children with fewer difficulties related to trauma, consistent with a study. ${ }^{17}$ This is not an issue, because the tool was designed to target children with a greater level of needs with regard to trauma. ${ }^{16}$ Surprisingly, the child strengths domain of the TC-CANS-Trauma showed an inadequate sensitivity, whereas that of the CANS-Trauma showed a satisfactory sensitivity. ${ }^{17}$ The difference might be attributed to cultural differences, as Hong Kong people tend to be critical when appraising positive information. ${ }^{39}$ Local clinicians are advised to adopt a more strength-based approach while assessing the competency and resources of Hong Kong traumatised children, adolescents, and young adults.

The current study has limitations. Owing to limited resources, a second assessor was not included and thus interrater reliability was not assessed, unlike similar validation studies that emphasise the communication value in the 
CANS-Trauma. ${ }^{18-20}$ Validation of the two optional domains (ratings for those aged $\leq 5$ years and those transitioning to adulthood) was not included. Inclusion of younger children and older adolescents may enable a more comprehensive evaluation of the characteristics of all ten domains as well as the age effects. Nonetheless, the findings of the study suggest the use of the eight domains of the TC-CANSTrauma for those aged 3 to 22 years given that the assessors provide the ratings "in the context of what is normative for the child's age/developmental stage", as stated in the guideline. ${ }^{16}$

\section{Conclusion}

The TC-CANS-Trauma is valid for the comprehensive assessment of trauma-related domains among Hong Kong children and adolescents. Its ratings can be used to guide the level of clinical intervention required. Clinicians are recommended to implement the TC-CANS-Trauma to facilitate trauma-informed practice in Hong Kong.

\section{Acknowledgements}

The authors would like to thank Hon-Wing Chu for initiating this validation project by first systematically searching existing validated scales and seeking consent from John $\mathrm{S}$ Lyons to translate the CANS-Trauma. Thanks also go to Yee-Ping Chung, Chor-Man Ma, Kwun-Yu Wong, and Lok-Yin Ho for their input in the translation procedure. I am grateful for the contributions of Polly Ho, Fabia Cheung, Miu Mau, Shannon Tsang, and Ella Leung for data input.

\section{Declaration}

The Boys' \& Girls' Clubs Association Jockey Club Trauma Treatment Service for Children was funded by The Hong Kong Jockey Club Charities Trust. The authors have no conflicts of interest to disclose.

\section{References}

1. Hopper EK, Bassuk EL, Olivet J. Shelter from the storm: traumainformed care in homelessness services settings. Open Health Serv Policy J 2010;3:80-100. Crossref

2. Berger R, Quiros L. Supervision for trauma-informed practice. Traumatology 2014;20:296-301. Crossref

3. Hendricks A, Conradi L, Wilson C. Creating trauma-informed child welfare systems using a community assessment process. Child Welfare 2011;90:187-205. Crossref

4. Cordell KD, Snowden LR, Hosier L. Patterns and priorities of service need identified through the Child and Adolescent Needs and Strengths (CANS) assessment. Child Youth Serv Rev 2016;60:129-35. Crossref

5. Spinazzola J, Ford JD, Zucker M, van der Kolk BA, Silva S, Smith SF, et al. Survey evaluates: complex trauma exposure, outcome, and intervention among children and adolescents. Psychiatr Ann 2005;35:433-9. Crossref

6. Ai AL, Foster LJJ, Pecora PJ, Delaney N, Rodriguez W. Reshaping child welfare's response to trauma: assessment, evidence-based intervention, and new research perspectives. Res Soc Work Pract 2013;23:651-68. Crossref
7. The Government of the Hong Kong Special Administrative Region. Epidemiology of child abuse and its geographic distribution in Hong Kong: an important social indicator of different districts and communities. Available from: https://www.pico.gov.hk/doc/en/ research_reports/CPU_research_report-epidemiology_of_child_ abuse_and_its_geographic_distribution_in_hong_kong.pdf

8. Ma EY, Li FW. Developmental trauma and its correlates: a study of Chinese children with repeated familial physical and sexual abuse in Hong Kong. J Trauma Stress 2014;27:454-60. Crossref

9. Lam LC, Wong CS, Wang MJ, Chan WC, Chen EY, Ng RM, et al. Prevalence, psychosocial correlates and service utilization of depressive and anxiety disorders in Hong Kong: the Hong Kong Mental Morbidity Survey (HKMMS). Soc Psychiatry Psychiatr Epidemiol 2015;50:1379-88. Crossref

10. Leung PW, Hung SF, Ho TP, Lee CC, Liu WS, Tang CP, et al. Prevalence of DSM-IV disorders in Chinese adolescents and the effects of an impairment criterion: a pilot community study in Hong Kong. Eur Child Adolesc Psychiatry 2008;17:452-61. Crossref

11. Food and Health Bureau, The Government of the Hong Kong Special Administrative Region. Mental Health Review Report. Available from: https://www.fhb.gov.hk/download/press_and_publications/ otherinfo/180500_mhr/e_mhr_full_report.pdf

12. Tang CSK. Educating for life adversities and challenges: mainstreaming trauma training in university and professional training curriculum. In: Wu KK, Tang CS, Leung EY, editors. Healing Trauma: a Professional Guide. Hong Kong University Press; 2011:15-34.

13. Wu KK. Trauma assessment tools validated in Hong Kong. In: Wu KK, Tang CS, Leung EY, editors. Healing Trauma: a Professional Guide. Hong Kong University Press; 2011:15-34.

14. Bernstein DP, Stein JA, Newcomb MD, Walker E, Pogge D, Ahluvalia $\mathrm{T}$, et al. Development and validation of a brief screening version of the Childhood Trauma Questionnaire. Child Abuse Negl 2003;27:16990. Crossref

15. Conradi L, Wherry J, Kisiel C. Linking child welfare and mental health using trauma-informed screening and assessment practices. Child Welfare 2011;90:129-47.

16. Kisiel C, Lyons JS, Blaustein M, Fehrenbach T, Griffin G, Germain J, et al. Child and Adolescent Needs and Strengths (CANS) Manual: the NCTSN CANS Comprehensive - Trauma Version: a Comprehensive Information Integration Tool for Children and Adolescents Exposed to Traumatic Events. Praed Foundation \& National Center for Child Traumatic Stress; 2010.

17. Kisiel C, Patterson N, Torgersen E, den Dunnen W, Villa C, Fehrenbach T. Assessment of the complex effects of trauma across child serving settings: measurement properties of the CANS-Trauma Comprehensive. Child Youth Serv Rev 2018;86:64-75. Crossref

18. Kisiel C, Blaustein M, Fogler J, Ellis BH, Saxe G. Treating children with traumatic experiences: understanding and assessing needs and strengths. In: Lyons JS, Weiner DA, editors. Behavioral Health Care: Assessment, Service Planning, and Total Clinical Outcomes Management. Civic Research Institute; 2009.

19. Anderson RL, Lyons JS, Giles DM, Price JA, Estle G. Reliability of the Child and Adolescent Needs and Strengths-Mental Health (CANSMH) Scale. J Child Fam Stud 2003;12:279-89. Crossref

20. Lyons JS. Communimetrics: a Communication Theory of Measurement in Human Service Settings. Springer; 2009. Crossref

21. Blake DD, Weathers FW, Nagy LM, Kaloupek DG, Gusman FD, Charney DS, et al. The development of a Clinician-Administered PTSD Scale. J Trauma Stress 1995;8:75-90. Crossref

22. Wu KK, Chan SK. Psychometric properties of the Chinese version of the Impact of Event Scale-Revised. Hong Kong J Psychiatry 2004;14:2-8.

23. Gray MJ, Litz BT, Hsu JL, Lombardo TW. Psychometric properties of the life events checklist. Assessment 2004;11:330-41. Crossref

24. Wu KK, Chan SK, Yiu VF. Psychometric properties and confirmatory factor analysis of the Posttraumatic Stress Disorder Checklist for Chinese survivors of road traffic accidents. Hong Kong J Psychiatry 2008; $18: 114-51$. 
25. Sclare I. The Child Psychology Portfolio. NFER-Nelson Publishing; 1997.

26. Wu KK, Chan SK, Hung OH, Cho VW. Psychometric properties and confirmatory factor analysis of the Children's Impact of Event ScaleRevised (CHIES-R) for child survivors of road traffic accidents in Hong Kong. Hong Kong J Psychiatry 2008;18:136-43.

27. Lau JT, Yeung NC, Yu XN, Zhang J, Mak WW, Lui WW, et al. Validation of the Chinese version of the Children's Revised Impact of Event Scale (CRIES) among Chinese adolescents in the aftermath of the Sichuan Earthquake in 2008. Compr Psychiatry 2013;54:8390. Crossref

28. Goodman R. The Strengths and Difficulties Questionnaire: a research note. J Child Psychol Psychiatry 1997;38:581-6. Crossref

29. Lai KY, Luk ES, Leung PW, Wong AS, Law L, Ho K. Validation of the Chinese version of the strengths and difficulties questionnaire in Hong Kong. Soc Psychiatry Psychiatr Epidemiol 2010;45:1179-86. Crossref

30. Lai KY, Leung PW, Luk ES, Wong AS. Use of the extended Strengths and Difficulties Questionnaire (SDQ) to predict psychiatric caseness in Hong Kong. Child Psychiatry Hum Dev 2014;45:703-11. Crossref

31. Gilmore L, Cuskelly M. Factor structure of the Parenting Sense of Competence scale using a normative sample. Child Care Health Dev 2009;35:48-55. Crossref
32. Johnston C, Mash EJ. A measure of parenting satisfaction and efficacy. J Clin Child Psychol 1989;18:167-75. Crossref

33. Ohan JL, Leung DW, Johnston C. The Parenting Sense of Competence scale: evidence of a stable factor structure and validity. Can J Behav Sci 2000;32:251-61. Crossref

34. Ngai FW, Chan WC, Holroyd E. Translation and validation of a Chinese version of the Parenting Sense of Competence Scale in Chinese mothers. Nurs Res 2007;56:348-54. Crossref

35. Linacre JM. Winsteps (version 4.4.5) Rasch Measurement Computer Program User's Guide. Available from: http://www.winsteps.com/

36. Horowitz M, Wilner N, Alvarez W. Impact of Event Scale: a measure of subjective stress. Psychosom Med 1979;41:209-18. Crossref

37. Layne CM, Greeson JKP, Ostrowski SA, Kim S, Reading S, Vivrette RL, et al. Cumulative trauma exposure and high risk behavior in adolescence: findings from the National Child Traumatic Stress Network Core Data Set. Psychol Trauma 2014;6(Suppl 1):S40-S49. Crossref

38. Thompson R, Lewis T, Neilson EC, English DJ, Litrownik AJ, Margolis B, et al. Child maltreatment and risky sexual behavior. Child Maltreat 2017;22:69-78. Crossref

39. Noguchi K, Gohm CL, Dalsky DJ, Sakamoto S. Cultural differences related to positive and negative valence. Asian J Soc Psychol 2007;10:68-76. Crossref 